Carnets de géographes

GÉOGRAPHES

\title{
Sociographie des lieux de culte musulman de Perpignan
}

Fouad Gartet et Rachid Id Yassine

\section{(2) OpenEdition}

Journals

Édition électronique

URL : http://journals.openedition.org/cdg/911

DOI : $10.4000 /$ cdg. 911

ISSN : 2107-7266

Éditeur

UMR 245 - CESSMA

Référence électronique

Fouad Gartet et Rachid Id Yassine, "Sociographie des lieux de culte musulman de Perpignan »,

Carnets de géographes [En ligne], 6 | 2013, mis en ligne le 01 septembre 2013, consulté le 19 avril 2019. URL : http://journals.openedition.org/cdg/911 ; DOI : 10.4000/cdg.911

Ce document a été généré automatiquement le 19 avril 2019

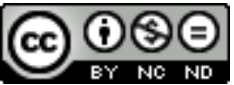

La revue Carnets de géographes est mise à disposition selon les termes de la Licence Creative Commons Attribution - Pas d'Utilisation Commerciale - Pas de Modification 4.0 International. 


\title{
Sociographie des lieux de culte musulman de Perpignan
}

\author{
Fouad Gartet et Rachid Id Yassine
}

\section{Introduction}

\section{L'islam en contexte sécularisé}

1 La vie religieuse en France est traditionnellement associée à l'expression du culte. Le droit français et la pratique de l'État laïc ont façonné une représentation de la religion à partir des manifestions cultuelles, seules autorisées et reconnues comme l'expression publique des religions. La notion de culte est centrale et participe à construire une conception générale du religieux réduite aux rites et cérémonies. La spiritualité se charge de véhiculer les autres formes plus subjectives de l'expérience religieuse. C'est là le pendant de la distinction entre sphère publique et sphère privée. En deçà de la pensée sociologique, c'est dans l'espace public de la société française, dans les rapports entre les acteurs politiques et religieux, que le culte s'est imposé comme l'ensemble des faits consensuels sur lesquels les politiques (les laïcs) et les religieux (les clercs) devaient négocier (Baubérot, 2010). Ces conditions générales du contexte national ont participé, à l'échelle locale, à l'installation de relations privilégiées entre le maire et les responsables des cultes.

2 Les pratiques religieuses - dans la mesure où elles se manifestent dans l'espace public qu'elles occupent physiquement par des bâtiments spécifiquement consacrés aux rites religieux (églises, synagogues, temples, mosquées...), des cimetières, des cérémonies religieuses (processions, festivités...) - impliquent un certain « interventionnisme » de l'État, étant notamment en charge d'assurer la sécurité et l'ordre publics. Contrairement aux instances religieuses, non seulement l'État laïc peut intervenir, aidé de la légitimité que lui confère le principe de neutralité au nom duquel il dit aussi agir, mais il le doit pour éviter qu'un pouvoir religieux ne règne souverainement sur tout ou partie des citoyens. 
3 Toutefois, « la laïcité est un mode particulier d'intervention religieuse (en fonction de choix religieux) impliquant de dénier la nature "religieuse" de l'intervention. Le plus souvent, les documents officiels n'évoquent que les "cultes" pour éviter d'évoquer les "religions" : sous prétexte de précision juridique, relevant en réalité d'une scolastique d'État, l'emploi de l'expression culte (ou "les cultes", la "pratique du culte", etc.) permet de "jouer sur les mots". Alors que définir ce qu'est la pratique du culte et ce qui ne l'est pas revient à tracer une frontière arbitraire, mais indiscutable, entre ce qui est digne d'être considéré comme une religion et ce qui ne l'est pas. Ces art(ifices) rhétoriques permettent encore d'éluder la question de la nature religieuse des politiques publiques laïques » (Liogier, 2006 : 26).

Ces remarques préalables nous permettent de comprendre pourquoi il importe d'abord de nous intéresser aux expressions cultuelles de l'islam, qui, dans le contexte singulier de la laïcité française, prennent une dimension centrale dans l'institutionnalisation de l'islam, et qui accordent une place de choix aux acteurs religieux en engageant une certaine cléricalisation de la religion musulmane. Il n'est donc pas anodin que l'instance mise sur pied, à la suite d'une succession d'initiatives d'organisation par le haut (Frégosi, 2008 : 222), fut nommée le « Conseil français du culte musulman » (CFCM). Or le culte n'est pas toute l'expression de l'islamité et c'est ainsi qu'émergea, " musulmans laïques contre religieux, la querelle de deux islams » (Frégosi, 2008 : 381), abstraction faite d'un troisième islam à la fois séculier et religieux, comme l'expression d'un « islam citoyen » (Venel, 2004) des nations européennes largement majoritaire.

\section{L'islam à l'épreuve de l'espace public local}

5 Mais à l'échelle locale, l'expression cultuelle de l'islam est dominante (Amiraux, 2012) ${ }^{1}$, comme l'illustre le cas du Roussillon où les associations musulmanes ne disposant pas de lieux de culte et menant des activités exclusivement culturelles, se comptent sur les doigts d'une main. Outre le recensement des espaces liés à la pratique du culte musulman, nous tâcherons ici d'en brosser un tableau à partir de données ethnographiques et urbanistiques. Leur croisement nous offre l'opportunité de mettre en évidence les articulations entre d'une part, le religieux en général et l'islam en particulier, et d'autre part, l'espace urbain d'une ville du Sud de la France.

Comme société sécularisée, la France offre une diversification de l'offre religieuse qui se traduit dans son paysage urbain de manière tout aussi disparate. Les lieux de culte participent à l'expression spatiale d'éléments marquants (symboles) dans les villes françaises, avec des typologies architecturales bien distinctes et qui résultent d'une conjugaison habile entre une "culture cultuelle $»^{2}$ spécifique et l'application juridique d'un règlement d'urbanisme. Nous verrons ici en quoi justement, il importe de penser la question des lieux de culte musulman à l'échelle de la ville. Par delà l'esprit d'uniformisation et la volonté de systématisation des textes légaux et réglementaires régissant l'implantation et la construction des bâtiments dédiés au culte, le développement des mosquées est soumis à des considérations sociales et politiques qui dépassent le strict cadre de l'administration de l'espace urbain et qui se cristallisent dans les relations entre le maire et les responsables des mosquées de la ville.

7 À travers le cas des mosquées de Perpignan, nous nous proposons d'étayer ici les modalités avec lesquelles la religion musulmane participe de l'émergence de nouvelles spatialités qui revisitent les représentations paysagères des villes européennes. Après une 
analyse descriptive du paysage religieux de l'islam perpignanais nous conduisant des dynamiques spatiales aux logiques sociales, nous examinerons les configurations du rapport entre spatialité et socialité que les mosquées de la ville dessinent.

\section{Les mosquées de Perpignan?}

\section{Penser la mosquée à l'extérieur du « monde musulman »?}

8 Lieu de rassemblement (aj-jama $a$ ) par excellence, la mosquée est certes un lieu de prosternation (as-sujûd) et un coin (az-zâwiya) de méditation. Toutefois, dans les villes arabo-musulmanes, la mosquée (du mot al-masjîd) a longtemps été aussi un lieu d'enseignement (religieux et séculier) où se situait la madrâsa, mais aussi un lieu de pouvoir (religieux et politique) et d'activités économiques se déroulant dans les échoppes, boutiques et marchés qui l'accolaient. Espace du sacré et du profane à partir duquel se marient donc le temporel et le spirituel, il structurait la vie sociale à l'échelle de la ville, la médina (Troin, 2001). Si les villes anciennes du Sud de la Méditerranée présentent des ressemblances et des formes urbaines homogènes, des plans spatiaux originaux et des bâtiments publics jouant un rôle majeur dans l'armature urbaine, c'est parce qu'elles étaient conçues par et pour l'homme, à travers le rôle central de la mosquée, dans le cadre d'une culture inspirée des préceptes de l'islam.

Aujourd'hui (temps) et à l'extérieur du « monde musulman » (espace), la mosquée semble toujours se prévaloir d'une capacité structurante pour un quartier, voire même pour l'armature spatiale communale. Participant à la vie sociale à l'échelle locale, elle apparaît comme un facteur d'intégration dans la mesure où les populations qui les fréquentent, majoritairement issues de l'immigration, y trouvent l'occasion d'exprimer la volonté de faire partie de la ville, a contrario du soupçon d'extranéité dont est habituellement l'objet l'islam. Agissant sur les interactions sociales, ces dynamiques induites par la présence d'une mosquée ont des incidences sur la configuration de nouvelles représentations de l'espace chez les musulmans certes, mais aussi chez l'ensemble des populations locales.

Dans le contexte de la France contemporaine, si le terme de mosquée est largement employé, dans le langage vernaculaire, pour désigner, plus techniquement, des ERP (Établissements Recevant du Public) dédiés aux pratiques religieuses des musulmans, l'expression de « lieu de culte musulman » nous est apparue comme celle qui convient le mieux pour nommer un bâtiment dédié essentiellement au rituel musulman de la prière ( as-salât). En Europe, l'hétérogénéité architecturale de ces lieux (caves, garages, appartements, maisons, villas, immeubles, bâtiments spécifiques) permet en effet à l'aspect fonctionnel d'apparaître comme le principal critère d'identification qui motive le recours à la dénomination de "mosquée ", sans tenir compte de la variété des appellations arabes qui distinguent notamment le jama', le masjî̀, la zâwiya, la musalla, la qấ'at-us-salât, etc.

11 Dans le cas de la ville de Perpignan, les lieux de culte musulman ont été généralement acquis à travers une opportunité foncière, sans que ne soit soulevée la question de la dénomination et à travers celle de la conception du projet. À l'exception de deux d'entre eux ayant fait l'objet d'une création neuve, ils sont le produit d'adaptation à un bâtiment ancien de type maison de ville sur plusieurs niveaux, ou de rénovation lourde, sans prise en compte des archétypes architecturaux des diverses cultures d'islam, et en particulier ici, ceux de la culture maghrébine. Certes, le rôle premier d'un architecte est de 
retranscrire les besoins d'un maitre d'ouvrage sur un lieu physique et social, mais il serait en la matière incongru d'importer ou de reproduire une architecture traditionnelle étrangère, tout comme il n'est guère satisfaisant d'imiter l'architecture autochtone selon une perspective assimilationniste. Il convient non seulement de tirer les leçons d'une organisation spatiale qui a bien fonctionné pendant des siècles dans les sociétés majoritairement musulmanes, mais aussi et surtout, de s'inspirer du patrimoine local pour s'y insérer dans une continuité féconde. La créativité inhérente à cette posture se retrouve dans la foisonnante production culturelle des musulmans des sociétés occidentales qui relève d'un éclectisme au principe des transformations identitaires en cours (Id Yassine, 2012 /1).

\section{Une typologie des lieux de culte musulman des villes occidentales}

12 Avec un jama, onze masâjid et une zâwiya, Perpignan et sa population de 117905 habitants ${ }^{3}$ disposent donc de treize lieux de culte musulman. L'étude détaillée de chacun des lieux de culte musulman de Perpignan nous a permis d'établir une typologie spatiofonctionnelle qui répond à la fois aux dispositions architecturales du bâtiment, aux modalités d'usage et de fréquentation, et à l'insertion dans le paysage urbain. Les résultats obtenus ${ }^{4}$ nous ont permis de mettre en évidence des similitudes et des distinctions.

En les confrontant à l'environnement urbain, ces données nous ont aidés à confirmer la typologie suivante. Nous avons ainsi pu distinguer trois types de lieu de culte musulman que nous appellerons " mosquée " autant par commodité que par fidélité au langage vernaculaire : 1) la mosquée intercommunale, 2) la mosquée inter-quartier et 3) la mosquée de proximité. En nous permettant d'observer, dans leur environnement urbain immédiat, trois lieux de culte musulman (mosquées de la Fraternité [13] ${ }^{5}$, Salam [5] et Malik [1]) caractéristiques de chacun de ces types de mosquée, les vues aériennes suivantes ${ }^{6}$ sont particulièrement démonstratives de la pertinence de cette typologie.

Figure . La mosquée intercommunale de l'agglomération de Perpignan

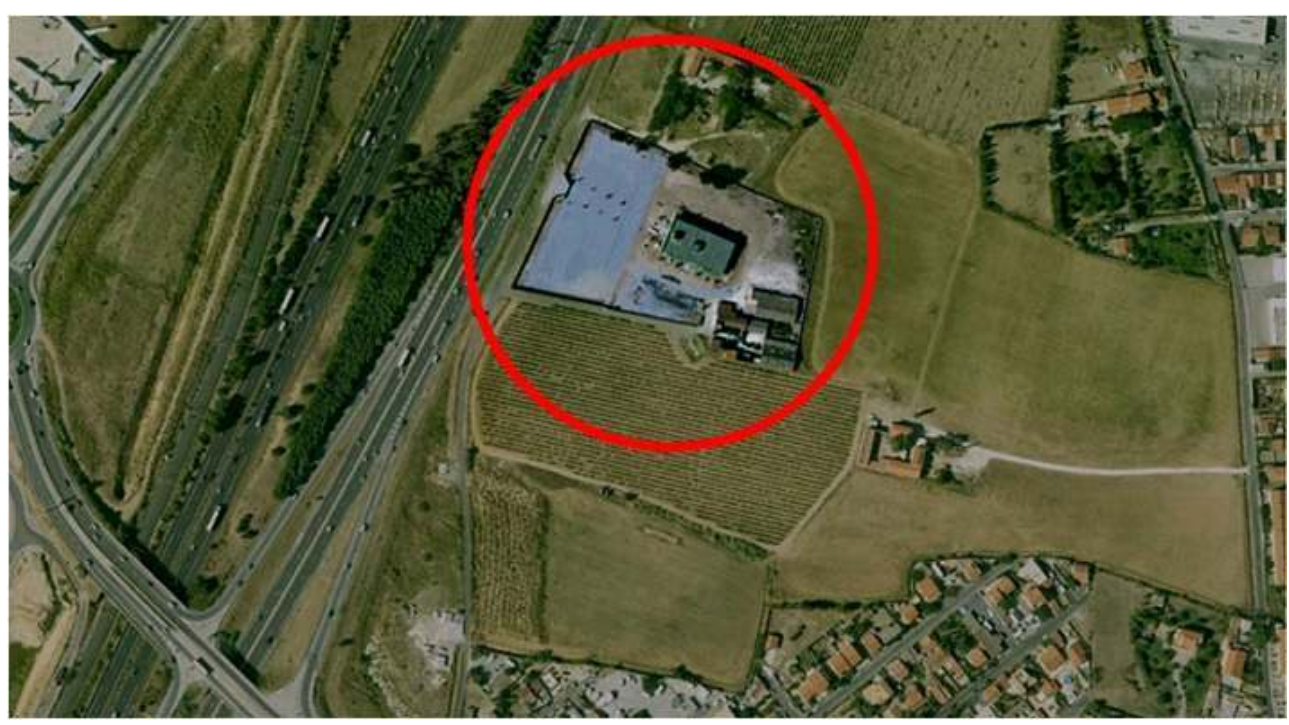

Un mitage urbain et une localisation spatiale marginalisée (cf. infra), malgré la volonté communale de reconstituer, à partir des grandes zones de logements HLM, l'ensemble d'un quartier, actuellement dégradé 
Figure . Une mosquée inter-quartier de Perpignan

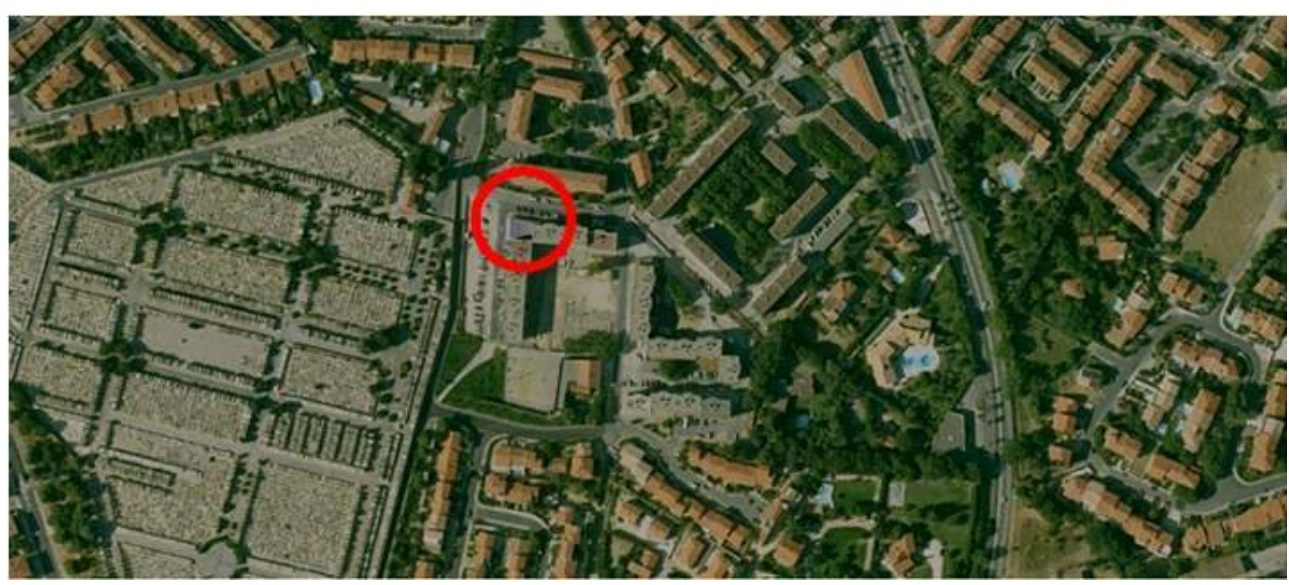

Le projet d'agrandissement en cours consiste en une surélévation d'un bâtiment existant dans un ensemble résidentiel "Cité des Rois de Majorque », de type "copropriétés dégradées », faisant partie des derniers projets réalisés par la commune dans le cadre du PNRU (Programme national de rénovation urbaine) dans l'objectif de rénover et requalifier le quartier.

Figure. Une mosquée de proximité de Perpignan

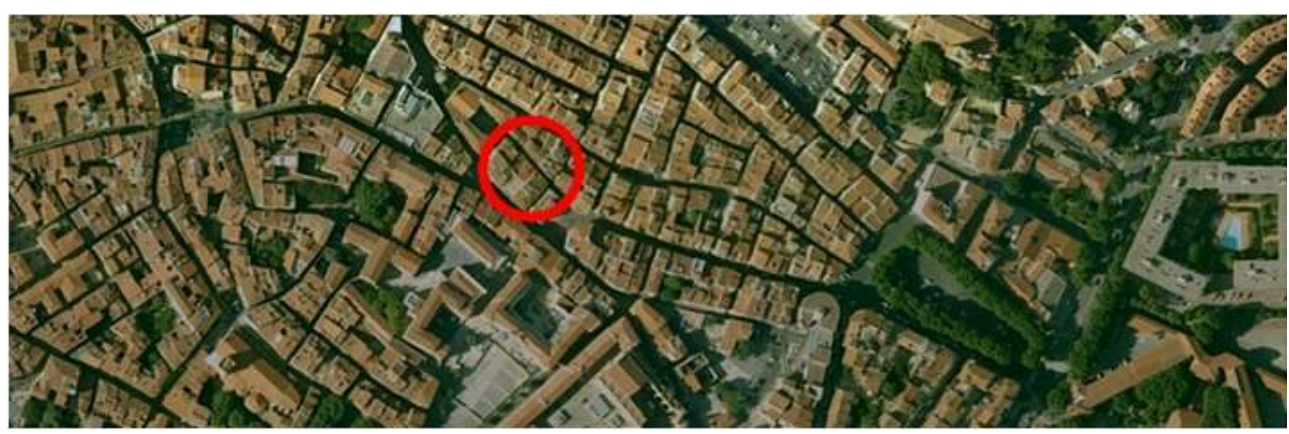

Le lieu de culte musulman est aménagé sur quatre niveaux dans une ancienne maison de ville. Située dans le centre ancien qui fait partie lui aussi du PNRU avec un projet d'éradication de l'habitat indigne dans une démarche de reconquête du centre ancien

14 L'implantation des lieux de culte musulman s'est fait au gré de l'aménagement du territoire urbain. Et si la politique de la ville a influé sur l'évolution et le développement des mosquées, la volonté municipale d'avoir un interlocuteur musulman à l'échelle de la ville et les compétences municipales en matière d'urbanisme, en particulier l'octroi de permis de construire et le droit de préemption, ont directement façonné le paysage cultuel musulman de Perpignan.

C'est alors que la mosquée de la Fraternité [13] se démarque clairement des autres lieux de culte musulman de la ville. Le bâtiment se situe en périphérie de la ville et est construit sur une parcelle lui permettant de disposer de sa propre aire de stationnement. À l'évidence, notamment du fait d'être excentré et situé à l'extérieur d'un quartier résidentiel, son rayonnement recouvre les communes avoisinantes. Comme pour plusieurs villes françaises et européennes, elle est « la mosquée de Perpignan » au sens à la fois politique ${ }^{7}$ et architectural de l'expression. Elle est donc la mosquée de type intercommunal du Roussillon. 
Figure . Plan du rez-de-chaussée de la mosquée intercommunale.

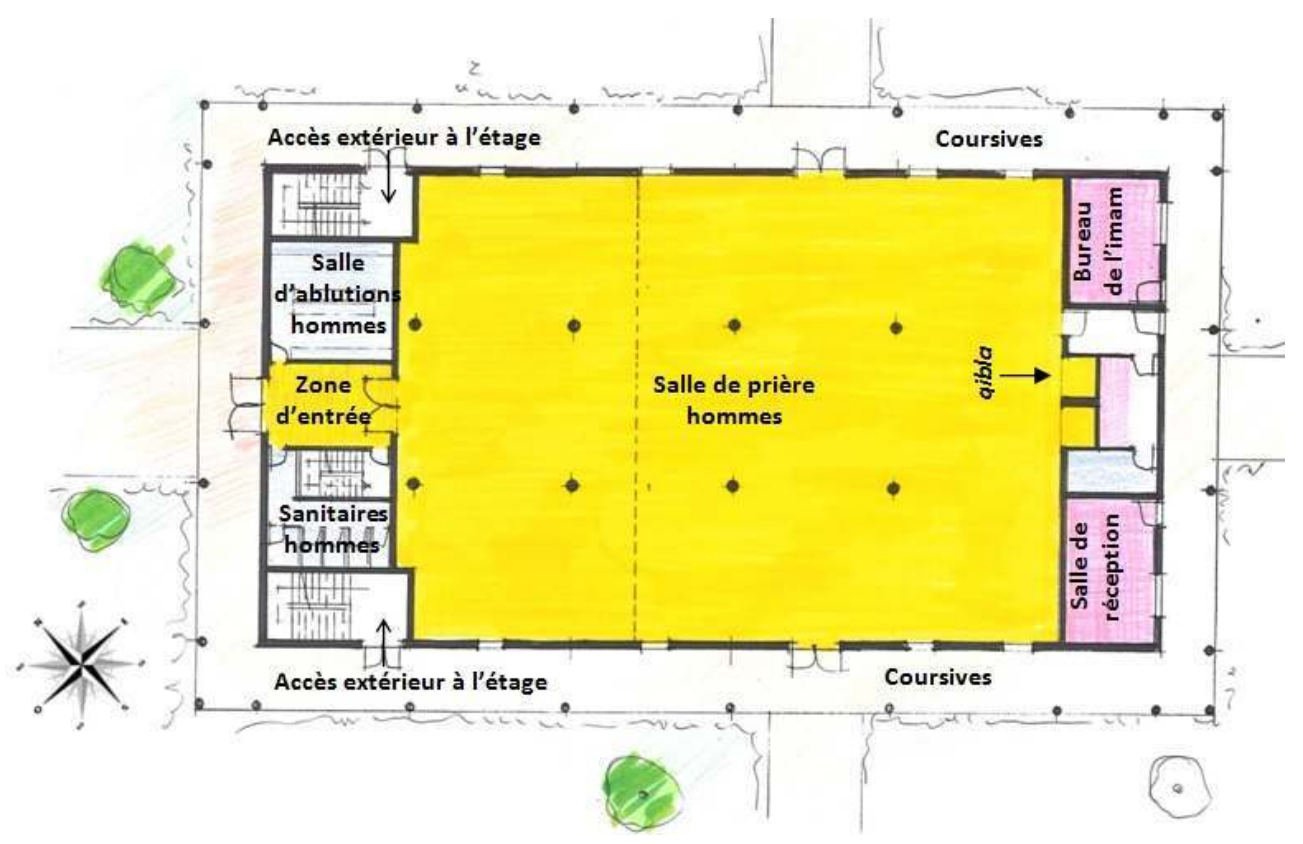

Le tissu spatial de cette zone est très diffus et la morphologie des bâtiments manque de cohérence. Il faut dire que la quasi-totalité des opérations immobilières de ce secteur ont été produites au coup par coup sans vision urbanistique stratégique préalable. La qualité architecturale des bâtiments s'en ressent. Aucune habitation ne se trouve donc à son immédiate proximité (voir la vue aérienne, Figure 1). Il est préférable de s'y rendre en véhicule, le trajet n'étant pas commode d'autant plus que le rite musulman de la prière se pratique à cinq moments de la journée, répartis de l'aube à la nuit tombée. Aussi la mosquée ne réunit-elle quotidiennement que quelques dizaines de fidèles pour un espace pouvant en accueillir plus d'un milliers.

Quatre autres lieux de culte musulman se distinguent par des caractéristiques urbaines, architecturales et fonctionnelles singulières : les mosquées Salam [5], Er-Rahma [7], ElFath [9] et Elhidaya [12]. Outre qu'elles accueillent toutes les fidèles pour la prière hebdomadaire, elles se démarquent des huit autres lieux de culte musulman de la ville par leur capacité d'accueil en cours de développement et/ou par leur fréquentation. Ces quatre bâtiments font en effet l'objet d'une extension ou d'une construction à la suite de l'acquisition foncière par leur association gérante. Situées au cœur de cités résidentielles, excepté la mosquée Er-Rahma derrière la gare ferroviaire, elles reçoivent des fidèles de plusieurs quartiers à la fois. 
Figure . Plan du rez-de-chaussée de la mosquée inter-quartier Salam.

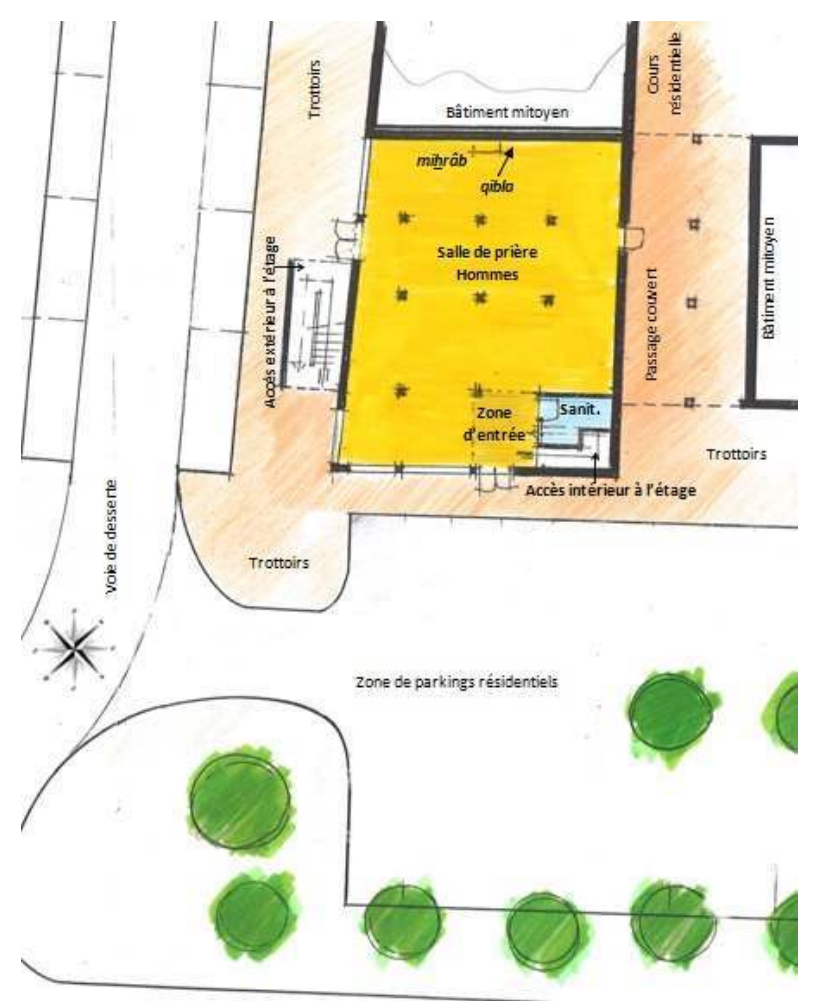

Le lieu de culte musulman géré par l'association Salam [5], dans le quartier de SaintMartin est représentatif des mosquées inter-quartiers implantées dans ces poches urbaines de logements sociaux. Sans pour autant ressembler aux ZUP du territoire national, ces zones en tâches d'huiles dans le paysage urbain perpignanais prennent la forme d'enclaves socio-spatiales. Le bâtiment en question est situé dans l'angle formé entre deux immeubles de la Cité des Rois de Majorque. Établi sur un seul niveau en rezde-chaussée, il est intégré à la composition paysagère du quartier. Le projet d'extension est rendu possible en surélevant le bâtiment, comblant ainsi le vide laissé dans l'angle. Le contexte urbain est celui d'une cité résidentielle qui a fait l'objet d'un plan de réhabilitation des façades et du cadre de vie extérieur, dans le cadre de l'ANRU (Agence nationale de rénovation urbaine).

Les autres lieux de culte sont ce que nous avons appelé des mosquées de proximité, accueillant la population résidente du quartier où elles se situent. Notons à ce propos qu'un projet d'agrandissement est à l'étude pour la mosquée Abou Bakr [6] qui, si la construction destinée à remplacer les préfabriqués actuels se réalise, verra le lieu de culte devenir une mosquée inter-quartier, d'autant plus que des fidèles des quartiers sud (Mas Bresson, Catalunya, Porte d'Espagne) et des villages au sud-ouest de Perpignan (Canohès, Toulouges, Thuir, Pollestres) la fréquentent déjà.

Ces huit lieux de culte de proximité sont notamment caractérisés par le fait que le bâtiment en question est antérieur à la conversion du lieu en mosquée. La mosquée des Turcs [8] (outre l'existence d'un projet de construction en cours) est une maison de ville. Celle du Champ-de-mars [4] est un local commercial tout comme l'a été la mosquée Salam [5] avant son actuelle réhabilitation. La salle de prière du Vernet Salanque [11] est un local en bas d'immeuble de la cité HLM du quartier. Le local de l'association Les sentiers 
[10] n'a pas subit de modification affectant le bâtit, et ne fut donc soumis qu'à une réfection de l'intérieur converti en zawiya. Enfin, les trois lieux de culte musulman du quartier Saint-Jacques [1, 2 et 3] sont à l'intérieur d'immeubles réaménagés en salles de prière.

Figure . Plan du rez-de-chaussée de la mosquée de proximité Malik.

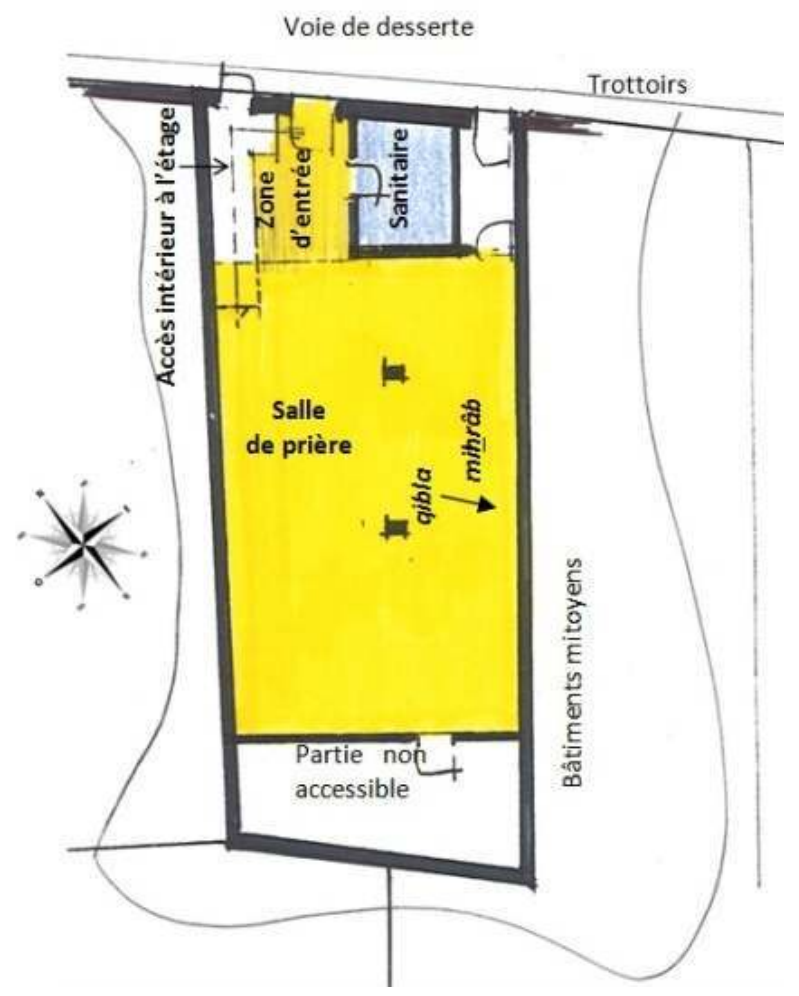

Parmi eux, la mosquée Malik [1] est caractéristique des mosquées de proximité du centre ancien de Perpignan. Situé en plein cœur de Saint-Jacques (voir la vue aérienne, figure 3), l'un des quatre quartiers du centre ancien de la ville, l'immeuble de trois étages est inséré dans un ensemble de bâtiments étroits. Placé en secteur sauvegardé, le quartier est caractérisé par l'insalubrité et fait l'objet d'une OPAH (Opération programmée d'amélioration de l'habitat). Le quartier classé en zone urbaine sensible est actuellement en pleine requalification.

Le paysage cultuel musulman perpignanais, du spatial au social. 


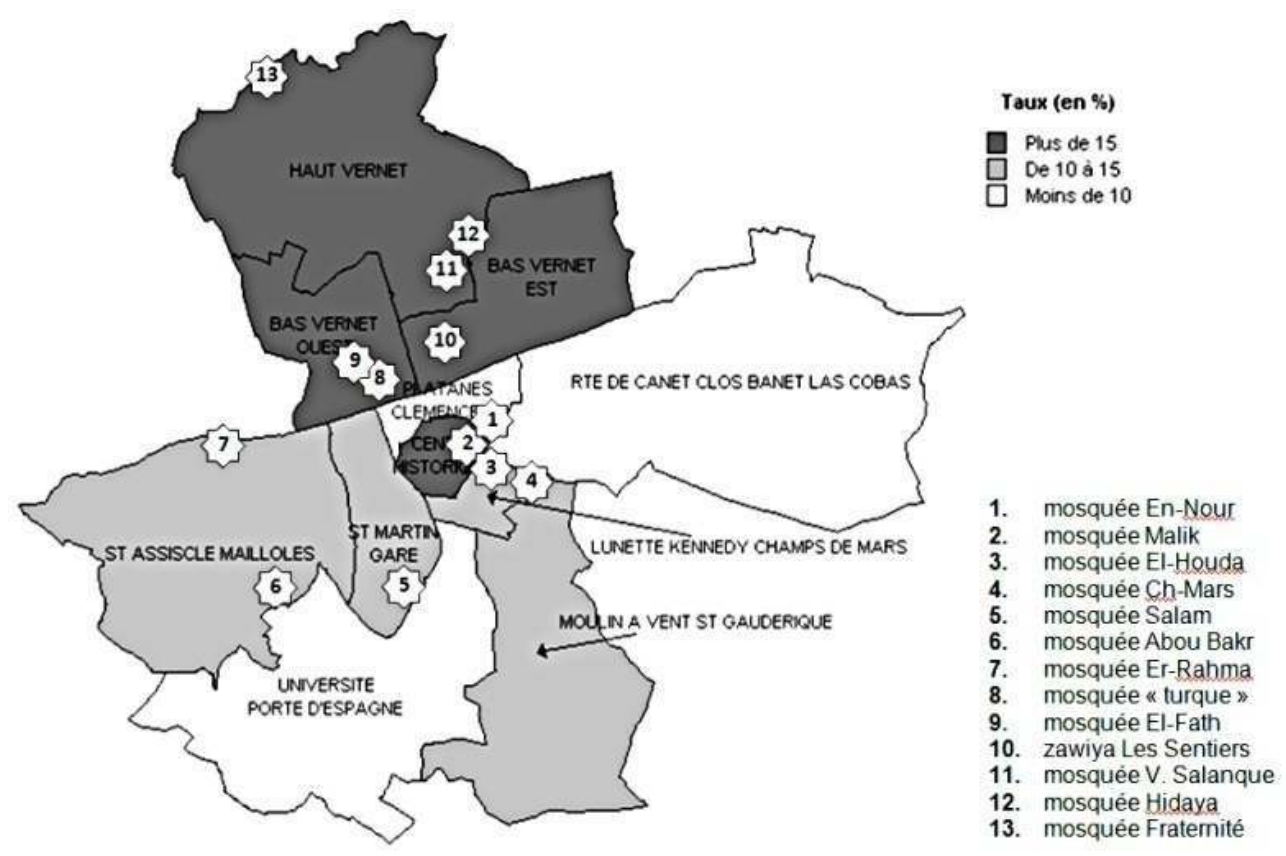

Sur la base d'une carte de l'INSEE indiquant la part des immigrés dans la population par quartier en 1999. Précision de l'INSEE : pour tenir compte des règles de secret liées aux variables sensibles, les cartes sont présentées par TRIRIS - regroupement d'au moins trois IRIS2000.

Crédits : Auteurs et INSEE - Recensement de la population @ IGN - INSEE 2003.

Tous les vendredis, des fidèles prient à l'extérieur des locaux de certaines mosquées qui assurent cet office religieux hebdomadaire. Ce qui alimente des plaintes pour « prières de rue » de la part de certains résidents. Rien d'étonnant si l'on s'en tient au fait qu'au moins 4300 musulmans se réunissent à l'occasion des prières annuelles. Avec une capacité maximale d'accueil des lieux de culte musulman de la ville de Perpignan de 3830 personnes dont 750 femmes, on pourrait conclure que les $2042 \mathrm{~m}^{2}$ de surfaces consacrées sur la ville à la prière musulmane seraient relativement suffisants. Or c'est au niveau de la répartition des fidèles qu'il convient d'apprécier la réalité de la situation.

On peut d'ailleurs s'apercevoir qu'il n'existe aucune démarche holistique à l'échelle de la ville. Ce défaut d'une prise en considération globale et cohérente de la question est imputable aussi bien aux décideurs politiques en matière d'aménagement du territoire qu'aux responsables associatifs musulmans. Ainsi en va-t-il de l'octroi de permis de construire qui n'obéit pas uniquement à des considérations objectives liées aux besoins concrets en matière de fréquentation des lieux de culte musulman. Si certains projets se justifient de ce point de vue (comme la mosquée Salam [5]), d'autres ne le sont guère (comme la mosquée Alhidaya [12]). Certaines mosquées ont un besoin urgent d'accroître leur capacité d'accueil, notamment celles du quartier Saint-Jacques pour lesquels des contraintes de réglementation en matière de sécurité se posent très sérieusement (degré de réaction au feu de certains matériaux, largeur des dégagements, nombre de sorties de secours, etc.). D'autres mosquées, dans le même cas de figure, disposent d'un espace extérieur qui leur permet de compenser l'insuffisance des superficies du bâti (mosquées Abou Bakr [6] et Al-Fath [9]). C'est ainsi qu'en réalité, même si la distribution des fidèles obéit à d'autres facteurs (proximité, mobilité, transport, rythme cultuel, attractivité de l'imam, orientation idéologique de l'association, activités annexes, etc.), la capacité 
d'accueil des mosquées de Perpignan satisfait mal le besoin des musulmans qui les fréquentent, et leur répartition sur le territoire communal n'est pas hiérarchisée.

Plus d'une décennie auparavant, d'aucuns voyaient dans « la communauté musulmane de Perpignan, quelque 10000 membres " (Feltin et Molénat, 11 octobre 2001). Avec aujourd'hui près d'un cinquième de ce chiffre qui assiste à la prière du vendredi - 2000 fidèles en moyenne sur l'année - il est indéniable que les difficultés dues à une prise en compte insuffisante des flux s'accentueront si aucune politique à l'échelle de la ville n'est mise en œuvre.

Le paysage cultuel musulman perpignanais met en évidence une configuration sociale et spatiale qui distingue les lieux de culte insérés dans des quartiers résidentiels à caractère social. La distinction s'avère nécessaire non seulement pour pouvoir mettre en relief les effets de lieux qui participent à la structuration du paysage religieux de la ville, mais aussi et surtout parce qu'elle souligne le rapport entre la mosquée et les caractéristiques sociodémographiques de la population musulmane. On peut également invoquer en faveur de ce choix, la capacité structurante du lieu de culte pour le quartier lui-même. ERP (établissement recevant du public) ${ }^{9}$, les lieux de culte animent par ailleurs la vie quotidienne du quartier, et contribuent à une relative « paix sociale $»^{10}$.

Cette prise en considération de la réalité sociodémographique liées à l'implantation des lieux de culte musulman permet donc de s'interroger sur l'existence outre d'un effet de classe, de mobilité ou de culture, d'un effet de lieu dans l'organisation des sociétés humaines, ou, si l'on préfère, de savoir si l'espace intervient comme facteur explicatif et isolable de l'organisation sociale et de quelle manière (Frémont et alii, 1984:387). Depuis les travaux pionniers de la géographie sociale, " on ne tente plus de détacher, d'isoler l'espace de l'organisation sociale, au contraire, on postule la consubstantialité du social et du spatial. On affirme d'emblée que la géographie étudie la dimension spatiale des phénomènes de société et qu'elle s'intéresse à l'organisation de l'espace et aux pratiques de l'espace des acteurs de la société ainsi qu'à leurs représentations ; ceci à différents niveaux de l'étendue, du local au mondial » (Sélimanovski, 2009/1:119) ${ }^{11}$.

Or si le lieu peut avoir un effet sur l'organisation sociale, il n'en demeure pas moins non plus qu'il est construit par les événements qui s'y déroulent. Dès lors, il nous appartient de rendre compte, au moment même où le lieu est présenté, des principaux événements qui participent de sa construction par les acteurs qui s'y trouvent. C'est à l'aide de l'imbrication de ces trois éléments, lieu, événement et acteur(s), que nous restituerons ici les modalités d'émergence de nouvelles spatialités qui concèdent à la religion une visibilité et un rôle fonctionnel réaffirmés dans la vie urbaine des sociétés européennes.

\section{Centre et marge, une question de perspective}

\section{La marginalité du centre ...}

28 Le paysage cultuel musulman de Perpignan est construit à partir de l'imbrication de facteurs sociaux et spatiaux. On peut déjà faire remarquer que les lieux de culte musulman de la ville sont tous situés dans des zones où la population immigrée est significativement présente (voir figure 7). Aucune des trois zones de la ville où celle-ci est inférieure à $10 \%$ de la population locale n'a de lieu de culte musulman. 

figure de centre "officiel » de la vie religieuse musulmane. Il s'agit de la mosquée intercommunale de notre typologie. En marge de la zone urbaine, elle a été construite dans la zone de Torremilla au Haut-Vernet, sur un terrain vague au milieu des vignes, en bordure de la voie rapide pénétrante à l'extrême nord de la ville, sur le bas-côté en face des hangars aéroportuaires, à proximité d'une zone commerciale et artisanale. Comme dans le cas de celui de « la mosquée à Mantes-la-Jolie », on retrouve à travers « la grande mosquée de Perpignan ", " les conditions imposées de l'hospitalité religieuse [à] un espace de relégation urbain et social [...] à une relative invisibilité sociale " (De Galembert, 2004 : 383-407). Le choix de cet emplacement avait fait l'objet de vives critiques de la part des fidèles peu motivés à participer à son financement initialement estimé à 610000 euros. Un financement bancaire, malgré l'interdiction religieuse du prêt à intérêts (ar-riba), aurait même été contracté pour faire aboutir le projet à son terme. « Il a [donc] fallu plus de 4 ans aux fidèles, qui ont versé pour cela quelque 900000 euros, pour réussir à construire ce qui constitue [en 2006] la troisième mosquée de France en termes de capacité cultuelle, derrière les mosquées de Paris et de Lyon » (La Gazette, 8 décembre 2006). Construit sur un terrain viticole en bordure de la voie rapide nord, principale axe routier qui longe lui-même l'autoroute, le bâtiment est donc un projet initial. Il est au centre du terrain sur lequel une aire de stationnement a été aménagée. S'étendant sur un terrain de $17500 \mathrm{~m}^{2}$, l'édifice dont ce fut « un architecte juif, Gérard Lascar, aujourd'hui disparu, qui a dressé gratuitement les plans » (Molénat, 2004) peut en effet se prévaloir d'être « la plus grande de France en terme de superficie » (L'Indépendant, 11 septembre 2010).

Le projet fut porté par feu A. Akkari. D'origine libanaise, ce chef du service orthopédie de l'hôpital Saint-Jean de Perpignan devint adjoint au maire délégué à la santé. En 2002, il fonda avec M. Iaouadan, avocat au barreau de la ville et également élu de la majorité municipale, le Collectif Fraternité Perpignan. Mais l'organisation est confondue avec le Collectif culturel et cultuel des musulmans des Pyrénées-Orientales qu'ils créèrent quatre ans plus tôt. Plus souvent appelé le Collectif des musulmans des Pyrénées-Orientales, il est aujourd'hui administré par trois médecins d'origine algérienne animant une troisième association fondée début 2006 et baptisée Centre Averroès Perpignan. Celle-ci est à vocation culturelle et permet ainsi d'obtenir des subventions publiques. Elle siège à l'angle sud-est du mas de la Gibelière, dans un ensemble de bâtis antérieurs à la construction de la mosquée servant aussi de résidence à l'imam. Ces trois associations de droit ne forment en réalité qu'une seule association de fait. On peut ainsi faire remarquer que médecins, avocats et élus locaux, ces responsables communautaires disposent des ressources 
sociales offrant une notoriété publique à la mosquée qui répondait d'ailleurs à un besoin plus politique que religieux.

En effet, maire de la ville de 1993 à 2009, J.-P. Alduy hérita de ce que le géographe Giband appelle :

« un système géopolitique spécifique : celui du clientélisme municipal, fondé sur le contrôle des positions de pouvoir local et un encadrement fort de la population selon un registre "ethnique" en faveur de deux groupes clairement identifiés à des territoires urbains [mais la différence du fils par rapport au père est qu'] à l'approche clientéliste, il substitue une dimension communautariste fondée sur l'appartenance religieuse » (2006/1:178).

Ce contexte et ces pratiques politiques locales ont largement participé à la configuration d'une représentation politique de la communauté musulmane qui plaça la « mosquée de la ville » au centre de l'espace public mais en marge de la vie communautaire. On retrouve ainsi à Perpignan les caractéristiques mises en évidence par Duthu (2008: 108) pour d'autres villes françaises : ancienneté du maire ; une "laïcité ouverte $»^{13}$ associée à une forte personnalité du maire qui atténue les postures identitaires et sécuritaires du parti politique d'affiliation (UMP) ; absence d'une coordination autonome des associations musulmanes à vocation cultuelle associée à l'absence paradoxale de relation avec le CRCM ${ }^{14}$ sensé assuré cette coordination ; clivage générationnelle avec une jeunesse musulmane coupée des responsables communautaires engagés dans un processus de notabilisation.

\section{... la centralité de la marge ...}

Dans le centre historique de la ville, Saint-Jacques porte les caractéristiques de ces " quartiers d'exil » dont parlent Dubet et Lapeyronnie (1999), avec 83 \% des habitants vivant sous le seuil de pauvreté.

« Le quartier Saint-Jacques est central au double titre de sa situation géographique et de son histoire. Il se présente comme un village de 800 immeubles étroits construits sur un site collinaire de 8 hectares, parcouru de ruelles de 3 à 4 mètres de large de structure labyrinthique, qui ne facilitent pas le passage des non-résidents. Soixante-dix pour cents des logements sont insalubres [...] Á l'hectare, la densité du bâti est de deux cents logements, et celle de la population est de cinq cent habitants » (Tarrius, 1999 : 57).

Parmi eux les musulmans du quartier sont essentiellement des personnes seules contraintes de rester sur le territoire national pour notamment continuer à percevoir leur retraite et autres droits sociaux. Les commerçants et les non-résidents forment l'autre composante des musulmans fréquentant l'un des trois lieux de culte de SaintJacques. Si deux d'entre eux [ 1 et 2$]$ bénéficient d'une antériorité historique, ces mosquées au centre de la ville sont en marge de la vie publique. Leurs responsables n'apparaissent aucunement dans l'espace public local.

Ces lieux de culte musulman ont été aménagés à l'intérieur d'anciens immeubles étroits, ils peuvent chacun accueillir près d'une centaine de fidèles sur plusieurs étages. Seuls des hommes les fréquentent, avec principalement des retraités marocains pour la mosquée En-Nour [1] et algériens pour la mosquée Malik [2]. Comme le laisse entendre la dénomination associative et comme le confirme l'enquête de terrain, l'association islamique cultuelle et apolitique prêche un islam traditionnel ne s'intéressant strictement qu'à la pratique des rituels religieux, ne dispensant aucune autre activité (éducative, sociale, culturelle...). 

trentenaires d'inspirations salafistes ${ }^{15}$, l'association $E l$ Houda (3) gère ce petit local situé derrière la rue Llucia, à deux pas du commissariat (de la Police nationale) du quartier. Commerçants de nationalité française, les responsables assurent un prêche du vendredi dans les deux langues (arabe et français), à près de 150 personnes qui remplissent la salle de prière. Ce chiffre tient compte du fait qu'en 2011 la salle a doublée sa capacité d'accueil avec une extension horizontale, en joignant deux locaux au rez-de-chaussée.

La distinction entre " espace matière » et " espace abstraction " permettant au sociologue Maurice Halbwachs d'empiler la réalité sociale (construite) sur des réalités matériels (physiques) nous offre dans le cas des mosquées de Saint-Jacques la possibilité d'examiner les «structures ou formes de la société [comme étant] par exemple, la façon dont se distribuent la population à la surface du sol » (Halbwachs, 1970 : 7). Cette distribution rend compte de la morphologie sociale d'un groupe à l'instar de la stratigraphie dans les sciences naturelles. «Le principe est que les structures sociales se matérialisent dans l'espace physique et inversement " (Beaubreuil, $2011: 160$ ) à telle enseigne que pour Halbwachs, si les groupes religieux se rattachent toujours à un « lieu central » physique et/ou symbolique, ils s'en détachent éventuellement.

Parlant de " géographie ou de topographie religieuse » (Halbwachs, 1997 : 231), il suggère ainsi que l'étude de la dimension spatiale des faits religieux porte notamment sur les représentations spatiales comme éléments de construction de l'espace social, et à terme de la vie sociale entière. Ainsi les communautés d'immigrés marocains et algériens ont édifié leurs lieux de culte comme espaces de socialisation matérialisant leurs conditions de migrants en marge de la scène publique locale. Cherchant aussi à se mettre à l'écart de la vie urbaine, les français salafistes de Perpignan se retrouvent, non plus par hasard, dans le même quartier à reproduire les conditions d'un imaginaire symbolique religieux en rupture avec la société immédiate, connecté à un univers sociopolitique hétérotope (Foucault, 1994 : 752).

\section{... et l'entre-deux}

Sept lieux de culte de Perpignan se situent donc à l'intérieur de quartiers HLM. Ils charrient une population dont les religiosités sont diverses, neutralisant ainsi un contrôle, voire même un monopole idéologique sur les activités qui s'y déroulent. Toutefois, cette diversité des affinités religieuses s'exprime au sein du sunnisme et illustre ainsi l'étonnante vitalité de ce que de nombreux sociologues appellent le " mouvement islamique $»^{16}$ contemporain. Elle participe, plus microsociologiquement, aux altercations régulières qui animent la vie des mosquées de Perpignan se situant à la frontière entre les espaces de la communauté et de la société.

41 Les mosquées insérées dans les cités où la population répond à des caractéristiques sociodémographiques diversifiées, sont le théâtre de vives interactions entre les fidèles. Ici les convertis s'opposent au « tribalisme berbère » ou au « nationalisme turc » ou les jeunes affrontent les plus âgés, tandis que là les femmes dénoncent « le machisme des responsables " ou les malékites font face aux wahhabites. Ces anicroches prennent rarement une tournure plus grave même si de violentes disputes apparaissent périodiquement. En tout état de cause, elles peuvent se ramener à une variété de conflits entre velléités progressistes des uns et aspirations conservatrices des autres. Dans tous les cas, la vie de ces mosquées est tributaire de l'espace dans lequel elles se trouvent à 
travers la variété des profils socioculturels des musulmans résidant dans ces quartiers populaires.

42 Autre caractéristique de ces lieux de culte, des activités éducatives extrascolaires s'y déroulent. En effet, les familles musulmanes de ces cités HLM délèguent à la mosquée du quartier l'instruction religieuse de leurs enfants et/ou l'accompagnement scolaire. Les responsables associatifs aménagent ainsi le lieu de culte de façon à assurer principalement des cours d'apprentissage de l'arabe et du Coran et de l'aide aux devoirs à plusieurs dizaines d'enfants qui se rendent au lieu de culte en soirée ou les matins de fin de semaine. En fait, seules les mosquées du quartier Saint-Jacques ne proposent pas d'activités pédagogiques, ce que l'exiguïté de l'immeuble et l'étroitesse des ruelles du quartier (l'insécurité nocturne) ne faciliteraient guère.

Autres activités qui s'y déroulent, les célébrations de mariage, baptême, décès et autres événements marquant de la vie familiale font l'objet de repas à l'intérieur de l'enceinte religieuse. À ces occasions, l'imam dispense quelques exhortations et des fidèles animent les soirées par des chants pieux. Les lieux sont dès lors équipés de cuisine improvisée permettant de préparer les dizaines de plats distribués à des fidèles présents sans nécessairement avoir été invités. Ces festivités entraînent parfois des nuisances nocturnes pour un voisinage qui très souvent s'en accommode.

Toutes ces activités et le nombre important de personnes qu'elles charrient, ont conduit à plusieurs projets de construction dans ces quartiers. La configuration des bâtiments et des alentours permet en effet des extensions nécessitant l'acquisition préalable de parcelles ou locaux. Tandis qu'une des mosquées du Bas-Vernet [8] et du Vernet-Salanque [11] et celle du Champs-de-Mars [4] envisagent d'agrandir leur lieu de culte, les cinq dernières années, les quartiers Saint-Martin [5], Bas-Vernet [9] et Vernet-Salanque [12] ont vu leur salle de prière se transformer en une mosquée pouvant accueillir plusieurs centaines de fidèles. Avec la rénovation de la mosquée Er-Rahma [7], c'est une croissance de plus de $21 \%$ de la capacité d'accueil de l'ensemble des lieux de culte de la ville que permettra l'ensemble des projets en cours de réalisation.

Rien d'étonnant donc à ce que ces quatre mosquées drainent une population bien au-delà de leur quartier d'implantation, entrainant des difficultés de circulation et de stationnement trop souvent négligées et sous-estimées ${ }^{17}$. Les représentations et les modes d'identification territoriale se trouvent affectés et se traduisent notamment dans les appellations vernaculaires des lieux de culte musulman identifiées au quartier ou à la cité 18. Ces lieux de culte vont jusqu'à participer à la structuration du quartier sans que cela ne soit pris en considération dans l'aménagement du territoire urbain. De véritables spatialités nouvelles apparaissent, faisant de certaines mosquées des lieux publics au sens technique du terme. Ces lieux doivent donc être pensés comme tels depuis leur aménagement jusqu'à leur usage ordinaire, car les incidences relatives à leur implantation dépassent leurs usagers pour concerner l'ensemble de l'espace urbain de manière aussi bien concrète (flux de véhicules, bruit, paysage urbain...) qu'abstraite (image de la ville, réputation du quartier...).

\section{Conclusion}

L'étude des relations entre les lieux de culte musulman de Perpignan et l'espace urbain de cette ville, nous a permis de mettre en évidence une interpénétration du spatial et du 
social. Celle-ci est d'autant plus probante que la verticalité de notre hiérarchisation spatio-fonctionnelle à travers la typologie des mosquées de Perpignan recoupe en partie l'horizontalité de notre descriptif socio-urbain. La configuration des dynamiques spatiales et sociales donne à voir leur articulation selon des modalités complexes dans la mesure où les facteurs sont hétérogènes. D'un côté l'analyse spatiale rend compte de trois types de mosquée qui reflètent autant les représentations que les pratiques de l'espace, de l'autre, l'analyse sociologique révèle trois situations différenciées en matière d'interactions entre communauté (religieuse) et société (civile).

La mosquée intercommunale, les quatre mosquées inter-quartiers et les huit mosquées de proximité construisent ainsi un paysage du culte musulman qui s'insère activement dans l'environnement urbain. Les acteurs et les événements, religieux et politiques, qui fondent ces lieux de vie sociale produisent des rapports à l'espace ambivalents. Ces derniers mettent en évidence une première centralité en marge de l'espace urbain et une seconde en marge de l'espace public local.

La mosquée intercommunale représente le centre politico-religieux de l'islam local. À travers un lieu architecturalement marqué, des évènements politiquement marquants comme la visite annuelle du maire de la ville à l'occasion de l'aïd, et des acteurs religieux engagés comme médiateur public entre la communauté musulmane et la société civile locales, la mosquée de la Fraternité s'impose comme la mosquée de la ville, celle qui occupe le centre de l'espace symbolique mais se situe à la périphérie de l'espace physique.

Les mosquées de proximité, quant à elles, elles se situent au cœur de la ville, et même pour certaines d'entre elles, dans le centre ancien. Elles témoignent également d'une certaine communautarisation des populations qui les fréquentent. Que l'on pense aux dynamiques ethno-nationales qui animent les mosquées des Turcs, des retraités marocains et algériens ou encore aux logiques de rupture avec la vie de la cité qui soustendent les mosquées salafi et soufi, ces mosquées de proximité offrent un portrait protéiforme du fait islamique à l'échelle locale, loin de cet essentialisme réifiant qui domine les représentations des communautés musulmanes.

Cette diversité s'illustre également à travers les mosquées inter-quartiers. On ne peut identifier ces lieux de culte musulman à une communauté ethnique, nationale ou idéologique particulière. Ils sont au contraire fréquentés par une population fortement hétérogène qui apprend à partager un même lieu où se vivent des religiosités différentes. Cette expérience du pluralisme religieux à l'intérieur d'une même famille spirituelle est en partie induite par des effets de lieux qui placent ces populations au cœur de la vie urbaine, dans les cités de la Cité. Ce pluralisme vécu font de ces mosquées des entre-deux, pas tout au fait au centre (ni spatial, ni social), les quartiers où elles se trouvent sont assimilés à la banlieue perpignanaise, mais pas non plus à la marge (ni spatiale, ni sociale), entre une communauté religieuse plurielle et une société locale dont elles participent au « vivre-ensemble ».

On le voit, la hiérarchisation des lieux de culte musulman sur un territoire est un élément majeur de la participation d'une population immergée de la ville, comme une composante à part entière de la vie urbaine. La réussite d'un projet de lieu de culte (financement, construction, gestion associative) passe également par l'implication des futurs usagers et dépend donc du degré d'adhésion des fidèles eux-mêmes, mais aussi de celui de la population de la ville en général (opposition d'une partie de l'opinion publique locale à la 
grande mosquée de Perpignan) et du quartier en particulier (rejet du projet de construction dans le quartier de Taialà à Gérone, commune voisine de Perpignan).

D'un point de vue opérationnel, il s'agit de développer de nouvelles méthodes de travail, de concertation et de coopération entre professionnels, responsables associatifs et institutionnels qui puissent tenir compte d'un processus de production participatif qui reformule les conditions d'un partenariat nécessaire qui passe notamment par de nouvelles formes de gouvernance des « projets collectifs». Les responsables associatifs gérant les lieux de culte musulman ne peuvent faire fi des incidences de diverses natures que produit la mosquée sur son environnement. Il convient notamment que les professionnels veillent à traduire les volontés des responsables associatifs dans une écriture architecturale cohérente, en harmonie avec les lieux avoisinants. Les décideurs politiques devraient mieux anticiper les conséquences de leurs décisions en matière d'urbanisme et de vie quotidienne de leurs administrés, musulmans ou non, électeurs ou non.

L'ensemble des acteurs engagés sont animés d'intérêts, de logiques et de visions loin de converger vers une stratégie partagée de l'occupation de l'espace urbain. Leurs interventions se répercutent aussi bien dans les pratiques que dans les représentations de l'espace de la ville. Comme sur d'autres projets, il serait bienvenu de systématiser des études d'impact, examinant la zone géographique d'influence du futur équipement religieux, élaborant la faisabilité technique du projet avant l'acquisition foncière (et préempter en connaissance de cause), articulant les bâtiments dans le tissu spatial : voies de communication, places de stationnement nécessaires au fonctionnement, espaces verts, etc. La ville est généralement structurée en quartiers fonctionnant entre eux. Ces derniers sont à leur tour structurés par les bâtiments et les infrastructures publics. Or sans être pour autant reconnu par les institutions nationales comme équipement public, nous avons vu à travers le cas de Perpignan que le lieu de culte musulman contribue, à sa hauteur, à la structuration du territoire des villes européennes d'aujourd'hui.

FELTIN M. et MOLÉNAT J. (11 octobre 2001) «L'islam modéré s'impose », lexpress.fr.

\section{BIBLIOGRAPHIE}

ALDUY J.-P. et OTAOLA J. (2004) Laïcité, spiritualités dans la cité, Perpignan : Ville de Perpignan.

AMGHAR S. (2011) Le salafisme aujourd'hui. Mouvements sectaires en Occident, Paris : Michalon.

AMIRAUX V. (2012) Chez soi en Europe : les musulmans à Paris (18 $\left.{ }^{\text {ème }}\right)$, Rapport de recherche. Londres : OSF.

BAUbÉROT J. (2010) Histoire de la laïcité en France, Paris : PUF.

BeAubreuil T. (2011) « Le "spatialisme" du dernier Halbwachs », Espaces et société, n¹44-145 « Usages populaires de l'espace », Toulouse : Érès. 
DE GALEMBERT C. (2004) « Le "oui" municipal à la mosquée à Mantes-la-Jolie : les faux-semblants de la reconnaissance de l'islam ", in GOTMAN A., Villes et hospitalité. Les municipalités et leurs "étrangers", Paris : Éditions de la Maison des sciences de l'homme.

DUBET F. et LAPEYRonNiE D. (1999) Les quartiers d'exil, Paris : Seuil.

DUTHU F. (2008) Le maire et la mosquée. Islam et laïcité en Île-de-France, Paris : L’Harmattan.

Foucault M. (1994) Dits et écrits 1954-1988, tome 4, Paris Gallimard.

FRÉGOSI F. (2008) Penser l'islam dans la laïcité, Paris : Fayard.

FREMONT A., HÉRIN R., CHEVALIER J., RENARD J. (1984) Géographie sociale, Paris : Masson.

GIBAND D. (2006/1) «Les événements de Perpignan ou la fin d'un système géopolitique local », Hérodote, $\mathrm{n}^{\circ} 120$.

GOT R. (2010) « Le "chaudron de la sorcière" : une lecture institutionnelle des "événements" de Perpignan », in olive J.-L., MUCCHIELli L. et GIBAND D., État d'émeutes, état d'exception. Retour à la question centrale des périphéries, Perpignan : Presses Universitaires de Perpignan.

HALBWACHS M. (1970) Morphologie sociale, Paris : Armand Colin.

HALBWACHS M. (1997) La mémoire collective, Paris : Albin Michel.

HERVIEU-LÉGER D. (2006) « La religion dans la constitution du lien social européen », in MICHALSKI K. (dir.), Conditions of European Solidarity. Vol. II. Religion in the New Europe, Budapest / New York : Central European University Press.

ID YASSINE R. (2012 /1) L'Islam d'Occident ? Introduction à l'étude des musulmans des sociétés occidentales, Perpignan : Halfa.

ID YASSINE, R. (2012 /2) Islam et régionalisme européen. Territoire, religion et identité en Catalogne française. Thèse de doctorat en sociologie, soutenue le 23 novembre 2012 à l'EHESS.

L'Indépendant (11 septembre 2010) «Fin du ramadan : l'Aïd el Fitr fêté par près de 3000 musulmans »

La Gazette (8 décembre 2006) «Inauguration de la grande mosquée de Perpignan », lagazettedescommunes.com

LIOGIER R. (2006) Une laïcité "légitime". La France et ses religions d'État, Paris : Médicis-Entrelacs. LUSSAULT M. (2007) L'homme spatial. La construction sociale de l'espace humain, Paris : Seuil. MOLÉNAT J. (2004) Le marigot des pouvoirs. Systèmes, réseaux, communautés, notables et francsmaçons en Languedoc-Roussillon, Castelnau-le-Lez : Climats.

NIEBUHR H. R. (1996) Christ and Culture, New York : Harper \& Row.

ROUGIER B. (dir.) (2008) Qu'est-ce que la salafisme?, Paris : PUF.

SÉLIMANOVSKI C. (2009/1), « Effets de lieu et processus de disqualification sociale. Le cas de Strasbourg et du Bas-Rhin », in EGGERICKX T., « Différences et inégalités socio-démographiques : approche par le local », Espace populations sociétés.

TARRIUS A. (1999) Fin de siècle incertaine à Perpignan. Drogues, pauvreté, communautés d'étrangers, jeunes sans emplois, et renouveau des civilités dans une ville moyenne française, Perpignan : Trabucaire.

TROIN J.-F. (2001) Les métropoles des « Sud », Paris : Ellipses. 
VENEL N. (2004) Musulmans et citoyens, Paris : PUF.

\section{NOTES}

1. Membre de l'équipe ayant réalisé l'enquête de terrain, nous avons pu observer cette même situation dans le cas de la ville de Paris, ce que Valérie Amiraux rapporte d'ailleurs dans son rapport en l'attribuant aux autorités politiques locales, et confortant par là nos remarques introductives : "La nouvelle équipe [municipale élue en 2001, écrit-elle] favorise une plus grande visibilité des musulmans dans la ville (permis de construire pour une mosquée, noms de rues, invitation de chefs spirituels musulmans à différentes cérémonies officielles, célébration du Ramadan comme une fête locale) et soutient les communautés musulmanes lors de la célébration des fêtes religieuses, par exemple en leur donnant accès à des gymnases pour y célébrer les prières du vendredi pendant le Ramadan et à l'occasion de l'Aïd el Kebir » (2012 : 72).

2. Ce qui ne va pas sans rappeler l'expression de « culture religieuse » (Niebuhr, 1996) ou encore celle de « religiosité culturelle » (Hervieu-Léger, 2006).

3. Insee, 2009, Recensement de la population.

4. Précision méthodologique : Pour l'ensemble des chiffres avancés, nous avons élaboré un modèle de calcul des capacités d'accueil maximales tenant compte des données architecturales, des pertes dues à l'incertitude de certaines informations, du nombre de fidèles... Les surfaces et les capacités d'accueil maximales sont calculées sur la base des bâtiments existant en juillet 2012 en tenant compte des chantiers en cours. Les fréquentations des lieux de culte ont été établies selon des fourchettes tenant compte des écarts importants entre les prières quotidiennes. Quant à la prière hebdomadaire du vendredi, il s'agit d'une moyenne des effectifs présents durant la période d'enquête (2009 à 2011), toujours arrondie au dixième.

5. Numéro renvoyant à la localisation sur la carte, figure 5.

6. Source : @ 2012, Google.

7. Cf. infra.

8. 900 hommes en rez-de-chaussée, 300 femmes à l'étage.

9. Le Code de la Construction et de l'Habitation prévoit a priori que les mosquées sont des ERP de type $\mathrm{V}$, allant à Perpignan de la catégorie 5 à 2 .

10. Il suffit de penser à Perpignan, au rôle de médiateurs que les imams ont joué au moment des émeutes de mai-juin 2005 (Id Yassine, 2012 /2 : 340).

11. Voir aussi Lussault (2007).

12. Les mosquées numérotés $4,5,6,8,9,11$ et 12 , voir figure 5 .

13. «Je suis un laïc qui ne refuse pas le spirituel » déclarait-il dans L'Indépendant du 29 janvier 2010. Voir l'ouvrage qu'il consacre à la laïcité (Alduy et Otaola, 2004).

14. Conseil régional du culte musulman, instance reliée au CFCM (cf. supra).

15. Voir notamment Rougier (2008) et Amghar (2011).

16. Voir son écho sur l'imaginaire européen ou le féminisme chez, respectivement, $\mathrm{N}$. Göle et S. Mahmoud. 
17. Le cas de la mosquée Er-Rahma située au bord d'une avenue est à ce titre exemplaire : l'association a été contrainte d'aménager une superficie considérable à l'intérieure du bâtit pour la destiner à un stationnement obligatoire.

18. Ainsi en est-il de " la mosquée de la Gare ", « la mosquée des Baléares ", «la mosquée de Mailloles », etc.

\section{RÉSUMÉS}

Recensant les espaces consacrés à la prière musulmane à Perpignan, cet article en offre un tableau descriptif à partir d'une enquête ethnographique et urbanistique. Le croisement des données obtenues met en évidence les articulations entre les singularités d'une pratique religieuse et l'espace urbain d'une ville du Sud de la France. Il permet de repenser la question des lieux de culte musulman non seulement à l'échelle de la ville mais aussi à l'extérieur du «monde musulman ». Outre les textes légaux et réglementaires régissant les bâtiments recevant du public, les mosquées sont soumises à des considérations sociales et politiques qui dépassent le strict cadre de l'administration de l'espace urbain et qui se cristallisent dans les relations entre le maire et les responsables des associations musulmanes de la ville.

Il apparaît très clairement que l'islam participe de l'émergence de nouvelles spatialités revisitant les représentations paysagères des villes européennes. L'étude des relations entre les lieux de culte musulman de Perpignan et l'espace urbain de cette ville montre une interpénétration du spatial et du social. En effet, la typologie des mosquées de Perpignan recoupe en partie la configuration socio-urbaine de la ville. D'un côté, l'analyse spatiale rend compte de trois types de mosquées qui reflètent à la fois les représentations et les pratiques de l'espace; de l'autre, l'analyse sociologique révèle trois situations différenciées en matière d'interactions entre communauté (religieuse) et société (civile). Dans la mesure où, notamment, la mosquée participe à la structuration du territoire des villes européennes, devrait-elle être considérée par les pouvoirs publics comme un équipement public à part entière?

Identifying the areas devoted to Muslim prayer in Perpignan, this article provides a descriptive table from an ethnographic and urbane investigation. Crossing data obtained highlights the links between the singularities of a religious practice and the urban space of a city in the South of France. It allows you to rethink the issue of Muslim places of worship not only at city level but also outside of the "Muslim world". In addition to laws and regulations governing public-access buildings, mosques are subject to social and political considerations which go beyond the strict framework of the administration of urban space and crystallize in the relationship between the mayor and officials Muslim associations of the city.

It is very clear that Islam contributes to the emergence of new spatiality revisiting the landscape representations of European cities. The study of relations between the Muslim places of worship of Perpignan and the urban area of this city demonstrates an interpenetration of space and society. Indeed, the typology of mosques of Perpignan partly collides with the socio-urban layout of the city. On the one hand, spatial analysis reports three types of mosque which reflect both the representations and practices of space; on the other, sociological analysis reveals three differentiated situations in terms of interactions between (religious) community and (civil) 
society. Insofar, especially, the mosque is taking part in the structuring of the territory of the European cities, should-it be considered by public authorities as a full-fledged public equipment?

INDEX

Thèmes : Carnets de recherches

\section{AUTEURS}

FOUAD GARTET

Architecte, urbaniste

archigaf@yahoo.fr

\section{RACHID ID YASSINE}

Docteur en sociologie de l'EHESS

rachidiy@ehess.fr 